\title{
AHSP: a novel hemoglobin helper
}

\author{
Arthur Bank
}

Department of Medicine and Department of Genetics and Development, Columbia University College of Physicians and Surgeons, New York, New York, USA.

\begin{abstract}
Recently, the small protein $\alpha$ hemoglobin-stabilizing protein (AHSP) was identified and found to specifically bind $\alpha$-globin, stabilize its structure, and limit the toxic effects of excess $\alpha$-globin, which are manifest in the inherited blood disorder $\beta$ thalassemia. In this issue of the JCI, Yu, Weiss, and colleagues show that AHSP is also critical to the formation and stabilization of normal amounts of hemoglobin, even when $\alpha$-globin is deficient, indicating unique and previously unidentified roles for this molecule (see the related article beginning on page 1856).
\end{abstract}

Normal human hemoglobin (Hb) formation and function is one of the marvels of human evolution and the process of natural selection. Get it right and you have the best functional molecule ever known to pick up oxygen in the lungs and deliver it to needy cells in order to keep their molecular and cellular engines operating at maximal efficiency. Get it wrong and there's big trouble.

Studies performed more than 50 years ago revealed the apparent relative simplicity of the process of $\mathrm{Hb}$ formation (Figure $1 \mathrm{~A}$ ). The $\alpha$-globin genes, of which two are present on each haploid chromosome 16, produce $\alpha$-globin polypeptides or chains (apo- $\alpha$ ); two $\beta$-globin genes, of which one from each parent is present on chromosome 11 , produce $\beta$-globin (apo- $\beta$ ). These $\alpha$ - and $\beta$-globin chains spontaneously combine with heme, an iron-containing moiety, to form $\alpha \mathrm{Hb}$ and $\beta \mathrm{Hb}$, respectively, which then dimerize as Hboß. The dimers then rapidly fold to form the functional oxygen-carrying tetramer $\mathrm{Hb}$, the major adult form being $\mathrm{HbA}$ (also known as $\mathrm{Hb} \alpha_{2} \beta_{2}$ ).

The assumption has been that the primary structure of the $\alpha$ - and $\beta$-globin chains encoded all of the information necessary for optimal folding and the production of the considerable amount of $\mathrm{HbA}$ critical for normal red cell function and survival. The tertiary structure of human $\mathrm{HbA}$ was predetermined by the primary amino acid sequence of the globin chains alone. No additional molecules or $\mathrm{Hb}$

Nonstandard abbreviations used: AHSP, $\alpha \mathrm{Hb}$-stabilizing protein; $\mathrm{Hb}$, hemoglobin.

Conflict of interest: The author has an equity interest in Genetix Pharmaceuticals Inc.

Citation for this article: J. Clin. Invest. 117:1746-1749 (2007). doi:10.1172/JCI32362. "helpers" were required for this to occur, or so we thought.

Evidence from earlier studies of the blood disorder $\beta$ thalassemia indicated that $\alpha$-and $\beta$-globin production by red cell precursors were independent processes. For example, in $\beta$ thalassemia, $\beta$-globin mutations result in reduced or absent $\beta$-globin synthesis in reticulocytes; however, $\alpha$-globin continues to be synthesized in normal amounts (1). The excess $\alpha$-globin either precipitates in erythroid precursors in the bone marrow (resulting in ineffective erythropoiesis), causing their premature destruction, or is proteolyzed (Figure 1B). Alternatively, the excess $\alpha$-globin binds to the cell membrane of adult erythroid cells, leading to their hemolysis (2). Essentially, disposition of excess $\alpha$-globin chains also appeared to occur without help from other molecules, despite causing some pathology along the way.

\section{Enter AHSP}

These processes seemed reasonable until Mitchell Weiss and colleagues described the first $\mathrm{Hb}$ "helper," $\alpha \mathrm{Hb}$-stabilizing protein (AHSP; also known as erythroidassociated factor [ERAF]), in $2002(3,4)$. AHSP is an abundant protein in red cells that binds tightly and specifically to apo$\alpha$-globin and $\alpha \mathrm{Hb}$ (Figure 1A). AHSP binds to two specific helices on $\alpha \mathrm{Hb}$ at overlapping sites in the same region that interacts with $\beta \mathrm{Hb}$. The $\alpha \mathrm{Hb}$-AHSP complex converts $\alpha \mathrm{Hb}$ to a unique, stable structure, dramatically altered in its 3D state, with its $\mathrm{Fe}$ ion configuration changing from $\mathrm{Fe}^{2+}$ to a more stable $\mathrm{Fe}^{3+}$ form (4-9). In the presence of sufficient concentrations of $\beta \mathrm{Hb}$, the greater affinity of $\alpha \mathrm{Hb}$ for $\beta \mathrm{Hb}$ compared with its affinity for AHSP facilitates the optimal "hand-off" of $\alpha \mathrm{Hb}$ from AHSP to $\beta \mathrm{Hb}$ and the subsequent formation of normal Hb $\alpha \beta$ dimers and HbA tetramers. AHSP does not react with apo- $\beta$-globin, $\beta \mathrm{Hb}$, or HbA tetramers.

Why is AHSP present in such high concentrations in normal erythroid cells: $0.1 \mathrm{mM}$ as compared with $5 \mathrm{mM}$ for $\mathrm{Hb}$ ? This concentration is certainly much higher than that necessary to interact with the small excess of $\alpha$-globin that accumulates in normal red cells. Indeed, it has been shown that mice devoid of AHSP (Absp $p^{-/}$ mice) have a mild anemia (3), presumably because the amount of apo- $\alpha$-globin and $\alpha \mathrm{Hb}$ is normally relatively small. It therefore appears that AHSP is not really criti$\mathrm{cal}$ in normal erythropoiesis in mice (and most of us) - we can get along without AHSP quite well.

New insights into the other potential roles of AHSP in red cells were obtained when Weiss et al. examined the effects of the loss of AHSP in a murine model of $\beta$ thalassemia (10). The anemia in these mice is much worse than that observed in either $A h s p^{-1-}$ mice without $\beta$ thalassemia or in mice with $\beta$ thalassemia and intact AHSP (10). The explanation for this observation is that AHSP normally stabilizes at least some of the excess of $\alpha$-globin produced in erythroid precursors in $\beta$ thalassemia by forming $\alpha \mathrm{Hb}$-AHSP complexes, rendering at least some of the excess $\alpha$-globin more soluble and stable and, therefore, causing less $\alpha$-globin precipitation and limiting ineffective erythropoiesis (Figure 1B). Apo- $\alpha$-globin and $\alpha \mathrm{Hb}$ are potential oxidants, especially in the absence of AHSP, generating ROS that lead to red cell membrane damage. AHSP provides some protection against these effects of excess $\alpha$-globin and $\alpha \mathrm{Hb}$. However, normal AHSP function is inadequate to stabilize the excess of $\alpha$-globin in $\beta$ thalassemia and prevent the severe anemia in mice or humans with the disease.

\section{New functions of the novel helper}

Now, in this issue of the JCI (11), Yu, Weiss, and colleagues show that AHSP is a much more adroit $\mathrm{Hb}$ helper, facilitating even more important new "twists" in $\mathrm{Hb}$ assembly. The authors show that AHSP is 
A Normal
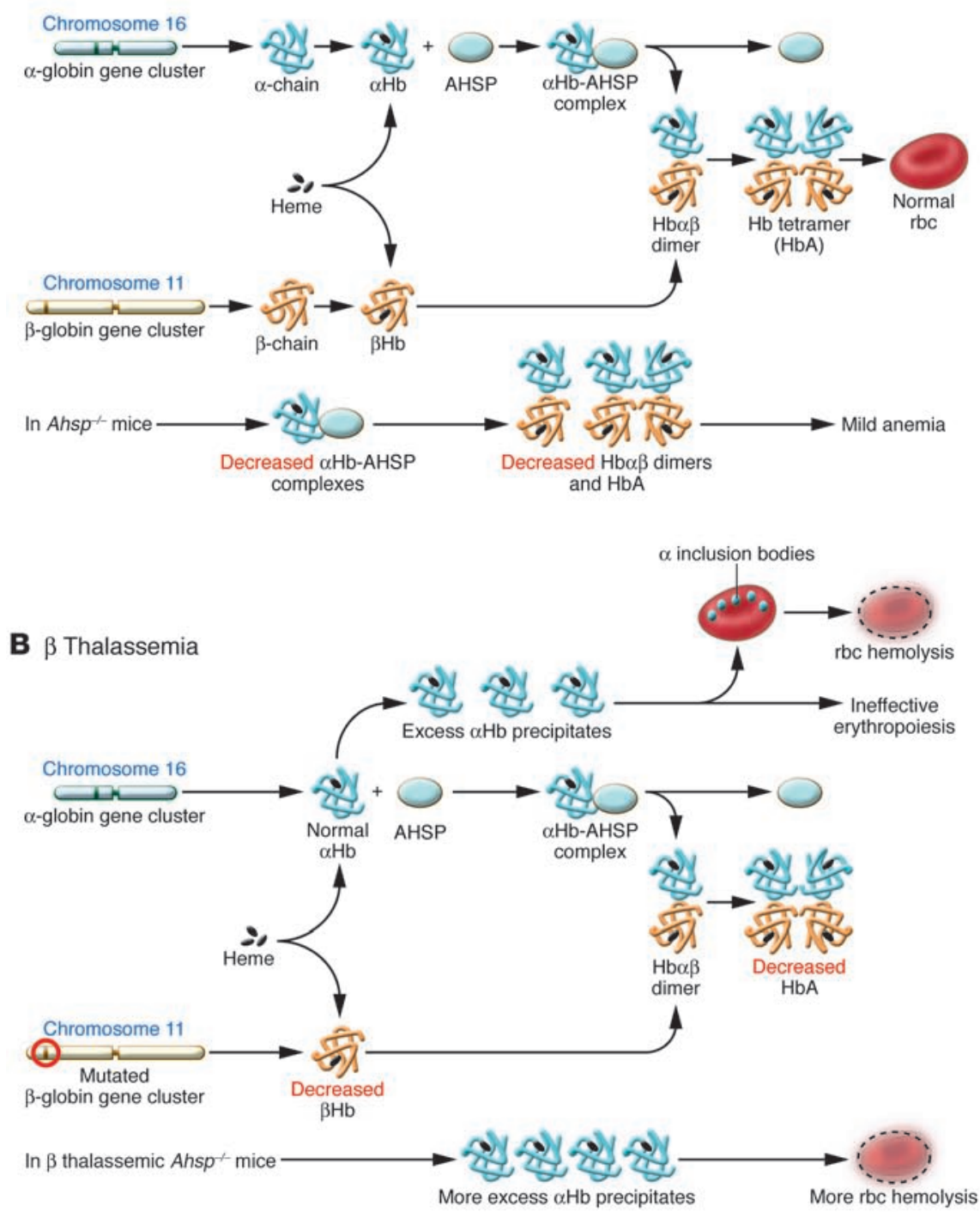

C $\alpha$ Thalassemia
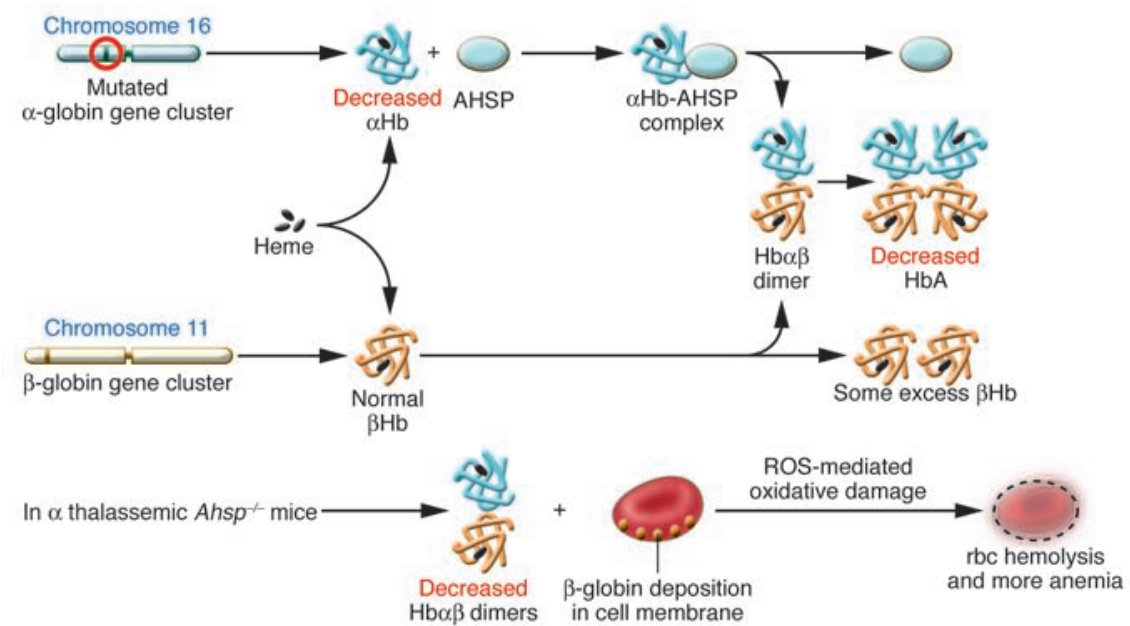

\section{Figure 1}

$\mathrm{Hb}$ formation with and without AHSP. (A) In normal cells, the $\alpha$-globin locus on chromosome 16 and $\beta$-globin locus on chromosome 11 produce $\alpha$ - and $\beta$-globin mRNA and $\alpha$ - and $\beta$-globin polypeptides, respectively, which combine with the heme moiety to form $\mathrm{Hb}$ dimers $(\mathrm{Hb} \alpha \beta)$. Two $\mathrm{Hb} \alpha \beta$ dimers combine and form the $\mathrm{Hb}$ tetramer $\mathrm{HbA}$. AHSP stabilizes and solubilizes newly formed and excess $\alpha$-globin chains as apo- $\alpha$-globin and $\alpha \mathrm{Hb}$. The absence of AHSP normally leads to a mild anemia due to precipitated unstabilized $\alpha$ chains (not shown). (B) In $\beta$ thalassemia, there is excess $\alpha$-globin formation due to decreased or absent $\beta$-globin production. The excess $\alpha$-globin precipitates in the cells and on membranes and leads to ineffective erythropoiesis in nucleated red cells in the bone marrow and red cell hemolysis in circulating blood cells. In the absence of AHSP, the anemia is worse because of the further destabilization of the excess $\alpha$-globin (not shown). (C) In this issue of the $\mathrm{JCl}$, Yu et al. (11) show that in mice with $\alpha$ thalassemia, there is an excess of $\beta$-globin chains due to a deficiency of $\alpha$-globin, and mild anemia. In $\alpha$ thalassemia in the absence of AHSP, $\beta$-globin precipitates in red cell membranes, presumably because the $\alpha$-globin-AHSP complex is a required intermediate for optimal $\mathrm{HbA}$ formation. 
important not only for dealing with newly synthesized excess $\alpha$-globin, but also in the assembly of normal $\mathrm{Hb}$ tetramers. In these new studies, $A b s p^{-1-}$ mice with mild $\alpha$ thalassemia were examined. This condition is associated with a deficit of $\alpha$-globin and an excess of $\beta$-globin, so no specific role for AHSP was expected to be required in these mice. However, $A b s p^{-/-}$mice with $\alpha$ thalassemia were found to be more anemic than either $A b s p^{-1-}$ mice or $\alpha$ thalassemic mice. If the role of AHSP is only to stabilize apo$\alpha$-globin and $\alpha \mathrm{Hb}$, why should its absence have any effect?

The authors provide some of the possible answers (11). They show that the anemia in the $A b s p^{-/-}$mice with $\alpha$ thalassemia is accompanied by an excess of precipitated $\beta$-globin chains in the membrane of erythroid cells (Figure 1C), which are present in much greater quantities than in either $A b s p^{-/-}$mice or $\alpha$ thalassemic mice. Thus, AHSP is required for the assembly of normally synthesized excess $\beta$ chains into functional $\mathrm{HbA}$ in these mice. Even the presence of small amounts of newly synthesized apo- $\alpha$-globin and $\alpha \mathrm{Hb}$ not stabilized by AHSP results in the inability of excess $\beta$-globin chains to be efficiently transferred into $\mathrm{HbA}$ in these $\alpha$ thalassemic mice. Thus, without AHSP, excess $\beta$-globin and $\beta \mathrm{Hb}$ are also unstable and deposited in the red cell membrane, leading to increased oxidative damage and more severe anemia (Figure 1C). The authors show that levels of ROS are greatly increased in the double mutants as compared with single mutants, reflecting this pathology (11). They also demonstrate that the deleterious effects of AHSP deficiency increase in marrow nucleated erythroid cell populations as they accumulate more globin and $\mathrm{Hb}$, confirming and extending previous results (12).

Potential roles for AHSP as an intermediate in the optimal formation of normal Hb $\alpha \beta$ dimers and/or tetramers remain to be determined. It is clear from this study (11), however, that AHSP is necessary for more than just chaperoning $\alpha$-globin around: it is also necessary for normal $\mathrm{HbA}$ assembly, especially when there is an imbalance in either $\alpha$ - or $\beta$-globin.

The results of several elegant in vitro experiments in this study (11) also provide new details regarding the way AHSP handles $\alpha$-globin in cells. Newly synthesized $\alpha$-globin chains, even nascent $\alpha$-globin chains on ribosomes, are rapidly complexed to AHSP in cell-free systems. More
$\mathrm{HbA}$ is formed in the presence of AHSP than without it. AHSP increases the resistance of apo- $\alpha$-globin to proteolysis by trypsin by promoting the proper folding of $\alpha$-globin in the $\alpha \mathrm{Hb}$-AHSP complex. Even after denaturation of apo- $\alpha$-globin chains, their renaturation is shown to be strongly promoted by the presence of AHSP (11).

\section{A role in red cell survival}

In summary, AHSP has been identified as a unique $\mathrm{Hb}$ helper, a molecular chaperone required for normal $\mathrm{Hb}$ assembly. $\mathrm{Yu}$, Weiss, and colleagues make the interesting suggestion that AHSP provides a selective advantage for the survival of red cells, especially when there are significant amounts of either excess $\alpha$ - or $\beta$-globin present (11). Interestingly, the red cells of patients with $\alpha$ and $\beta$ thalassemia are more resistant to the severe form of malaria than normal cells (13). The evolution of AHSP may have permitted the preferential survival of these cells. Without AHSP, the thalassemic red cells might not have survived, while with it, they are able to. Thus, AHSP may have evolved to give erythroid progenitors an "edge," especially when mutations occur that lead to significantly unbalanced $\alpha$ - or $\beta$-globin levels. Then, throughout evolution, the AHSP-expressing cells with globin mutations may have been further selected to survive because these cells prevented fatal malarial infection.

Also, because of its effects on preventing $\alpha$-globin denaturation and promoting renaturation, AHSP may provide an additional selective advantage to red cells under conditions of oxidative stress induced by drugs that cause a greater susceptibility to hemolysis. AHSP may also be useful to red cells in iron deficiency in which heme availability is limited and apo$\alpha$-globin levels are increased. These functions may represent additional evolutionbased roles for AHSP in the stabilization of red cells in the presence of environmental factors that alter Hb's critical equilibrium. No human disease resembling AHSP deficiency has yet been described, although associations between the severity of $\beta$ thalassemia in patients with variations in AHSP are being explored $(14,15)$. In a case of a naturally occurring human $\alpha$-globin chain mutation, in which the binding site for $\alpha \mathrm{Hb}$-AHSP complex formation is altered, there is decreased stability of the resulting human $\mathrm{Hb}$ (16).
Are there other Hb helpers, be they specific $\alpha$-globin chaperones or other erythroidspecific or ubiquitous molecules, with which AHSP interacts? It is known that the transcription factors GATA-1, OCT-1, and EKLF are required for AHSP expression $(17,18)$. How does AHSP interact with these and other transcription factors and intermediates affecting heme biosynthesis and posttranscriptional modifiers in red cells in the process of Hb synthesis and assembly? These and other questions regarding our understanding of $\mathrm{Hb}$ regulation remain. We are also still in the dark about what controls the differentiation of nucleated red cells and their enucleation, what regulates the filling of red cells with the desired amount of $\mathrm{Hb}$, and how that amount is maintained until red cell death. Does AHSP function in these events? Weiss et al. (11) have given us a start by identifying an important $\mathrm{Hb}$ helper, but there is plenty of room for researchers to discover other $\mathrm{Hb}$ helpers and to shed more light on this subject.

Address correspondence to: Arthur Bank, Department of Genetics and Development, Columbia University College of Physicians and Surgeons, Armand Hammer Health Science Center, HHSC 16-1604, 701 West 168th Street, New York, New York 10032, USA. Phone: (212) 3054186; Fax: (212) 923-2090; E-mail: ab13@ columbia.edu.

1. Bank, A., Braverman, A.S., O'Donnell, J.V., and Marks, P.A. 1968. Absolute rates of globin chain synthesis in thalassemia. Blood. 31:226-233.

2. Rachmilewitz, E., and Schrier, S. 2001. Pathophysiology of $\beta$ thalassemia. In Disorders of hemoglobin. R. Nagel, editor. Cambridge University Press. Cambridge, United Kingdom. 233-251.

3. Kihm, A.J., et al. 2002. An abundant erythroid protein that stabilizes free $\alpha$-haemoglobin. Nature. 417:758-763.

4. Gell, D., Kong, Y., Eaton, S.A., Weiss, M.J., and Mackay, J.P. 2002. Biophysical characterization of the $\alpha$-globin binding protein $\alpha$-hemoglobin stabilizing protein. J. Biol. Chem. 277:40602-40609.

5. Feng, L., et al. 2004. Molecular mechanism of AHSP-mediated stabilization of $\alpha$-hemoglobin. Cell. 119:629-640.

6. Baudin-Creuza, V., et al. 2004. Transfer of human $\alpha$ to $\beta$-hemoglobin via its chaperone protein: evidence for a new state. J. Biol. Chem. 279:36530-36533.

7. Santiveri, C.M., et al. 2004. NMR structure of the $\alpha$-hemoglobin stabilizing protein: insights into conformational heterogeneity and binding. J. Biol. Chem. 279:34963-34970.

8. Feng, L., et al. 2005. Structure of oxidized $\alpha$-haemoglobin bound to AHSP reveals a protective mechanism for haem. Nature. 435:697-701.

9. Hamdane, D., Vasseur-Godbillon, C., Baudin-Creuza, V., Hoa, G.H., and Marden, M.C. 2007. Reversible hexacoordination of $\alpha$-hemoglobin-stabilizing protein (AHSP)/ $\alpha$-hemoglobin versus pressure. 
Evidence for protection of the $\alpha$-chains by their chaperone. J. Biol. Chem. 282:6398-6404.

10. Kong, Y., et al. 2004. Loss of $\alpha$-hemoglobin-stabilizing protein impairs erythropoiesis and exacerbates $\beta$-thalassemia. J. Clin. Invest. 114:1457-1466. doi:10.1172/JCI200421982

11. Yu, X., et al. 2007. An erythroid chaperone that facilitates folding of $\alpha$-globin subunits for hemoglobin synthesis. J. Clin. Invest. 117:1856-1865. doi:10.1172/JCI31664.

12. dos Santos, C.O., Duarte, A.S., Saad, S.T., and Costa, F.F. 2004. Expression of $\alpha$-hemoglobin stabilizing protein gene during human erythropoiesis. Exp. Hematol. 32:157-162.
13. Senok, A.C., et al. 1997. Invasion and growth of Plasmodium falciparum is inhibited in fractionated thalassaemic erythrocytes. Trans. R. Soc. Trop. Med. Hyg. 91:138-143.

14. Viprakasit, V., et al. 2004. Evaluation of $\alpha$ hemoglobin stabilizing protein (AHSP) as a genetic modifier in patients with $\beta$ thalassemia. Blood. 103:3296-3299.

15. Lai, M.I., et al. 2006. $\alpha$-Haemoglobin stabilising protein is a quantitative trait gene that modifies the phenotype of $\beta$-thalassaemia. Br. J. Haematol. 133:675-682.

16. Vasseur-Godbillon, C., Marden, M.C., Giordano, P., Wajcman, H., and Baudin-Creuza, V. 2006
Impaired binding of AHSP to $\alpha$ chain variants: $\mathrm{Hb}$ Groene Hart illustrates a mechanism leading to unstable hemoglobins with $\alpha$ thalassemic like syndrome. Blood Cells Mol. Dis. 37:173-179.

17. Gallagher, P.G., Liem, R.I., Wong, E., Weiss, M.J., and Bodine, D.M. 2005. GATA-1 and Oct-1 are required for expression of the human $\alpha$-hemoglobin-stabilizing protein gene. J. Biol. Chem. 280:39016-39023.

18. Pilon, A.M., et al. 2006. Alterations in expression and chromatin configuration of the $\alpha$ hemoglobin-stabilizing protein gene in erythroid Kruppel-like factor-deficient mice. Mol. Cell. Biol. 26:4368-4377

\title{
Torn apart: membrane rupture in muscular dystrophies and associated cardiomyopathies
}

\author{
Jan Lammerding and Richard T. Lee
}

Cardiovascular Division, Department of Medicine, Brigham and Women's Hospital, Harvard Medical School, Boston, Massachusetts, USA.

\begin{abstract}
Muscular dystrophies are often caused by mutations in cytoskeletal proteins that render cells more susceptible to strain-induced injury in mechanically active tissues such as skeletal or cardiac muscle. In this issue of the JCI, Han et al. report that dysferlin participates in membrane resealing in cardiomyocytes and that exercise results in increased membrane damage and disturbed cardiac function in dysferlin-deficient mice (see the related article beginning on page 1805). Thus, in addition to repetitive membrane damage, inadequate membrane repair may participate in the pathogenesis of muscular dystrophies and cardiomyopathies.
\end{abstract}

Given the large deformations of many tissues such as skeletal muscle, it's remarkable that cells don't just tear apart. In fact, membrane rupture occurs frequently under physiological conditions in mechanically active tissues and can be experimentally demonstrated both in vivo and in vitro (1-3). Membrane rupture leads to loss of cytoplasmic components and allows the influx of extracellular ions, thus disturbing the carefully maintained ion balance between the cytoplasm and the extracellular space. To prevent cell death from local membrane damage, cells have mechanisms to preserve membrane integrity and to reseal or repair ruptured membranes. In the presence of high extracellular $\mathrm{Ca}^{2+}$ concentrations, injured nucleated cells reseal within 10-30 seconds (3).

\section{Membrane-repair mechanism(s)}

Membrane rupture leads to exposure of hydrophobic phospholipids to the aque-

Conflict of interest: The authors have declared that no conflict of interest exists.

Citation for this article: J. Clin. Invest. 117:1749-1752 (2007). doi:10.1172/JCI32686. ous environment, an energetically unfavorable state. However, the entropic forces that draw the membrane ends together are insufficient to reseal membrane lesions larger than $1 \mu \mathrm{m}$ in nucleated cells under physiological conditions (3), as membrane tension (driven by interaction of phospholipids with the underlying cytoskeleton) slows or completely blocks self sealing. Instead, cells utilize an active membrane-repair process based on active trafficking of endomembrane vesicles to the damage site and subsequent fusion with the plasma membrane by exocytosis, but many of the molecular details of this process remain unclear. It appears that membrane repair involves both a reduction in membrane tension - possibly by local depolymerization of the cortical cytoskeleton - and patch formation. In the latter process, homotypic fusion of membrane vesicles creates a patch at the rupture site that then fuses with the plasma membrane in a $\mathrm{Ca}^{2+}$-dependent process (Figure 1). Depending on the cell type, the vesicular membrane compartments participating in the repair may include cortical granules, yolk granules, endocytic components, lysosomes, and enlargosomes; the specific contribution of each is not always clear $(3,4)$.

The active membrane fusion process requires several membrane proteins, including SNARE proteins (a family of transmembrane proteins essential in most intracellular membrane fusion processes) and synaptotagmins (transmembrane proteins containing two highly conserved $\mathrm{Ca}^{2+}$-binding domains that are thought to serve as $\mathrm{Ca}^{2+}$ sensors; ref. 5). Most recently, ferlins have been identified as a conserved protein family that participate in membrane repair. The ferlin family consists of four different genes that encode dysferlin, myoferlin, otoferlin, and Fer1L4; dysferlin-null cells show accumulation of membrane vesicles near the damaged membrane $(3,4)$.

\section{Membrane disruption leads to muscular dystrophy}

Membrane rupture is most prevalent in skeletal and cardiac muscle; up to $25 \%$ of muscle cells show signs of transient membrane damage under physiological conditions (1-3). Higher organisms have specialized tissue and cell architectures that minimize the stress placed on the plasma membrane by directly transmitting forces from the extracellular matrix to the cytoskeleton through dedicated protein complexes, such as the dystrophin-glycoprotein complex, and thus shielding the fragile plasma membrane. Not surprisingly, mutations that disrupt these stress-bearing elements render cells 